\title{
Capsule Commentary on Hunderfund et al., Imprinting on Clinical Rotations: Multisite Survey of High- and Low-Value Medical Student Behaviors and Relationship with Healthcare Intensity
}

Daniel G. Hoody, MD, MS

Academic Hospital, Hennepin Healthcare, Minneapolis, MN, USA.

J Gen Intern Med 34(7):1290

DOI: $10.1007 / \mathrm{s} 11606-019-04905-\mathrm{y}$

(c) Society of General Internal Medicine 2019

$\mathrm{H}$ underfund et al. ${ }^{1}$ explored imprinting on medical trainees by comparing medical student value behaviors to regional health care intensity with a survey that measured high-value and low-value medical student behaviors. They found that while most students claimed both high- and lowvalue behaviors, there was a statistically significant correlation between low-value behaviors and intensity but not between high-value behaviors and intensity.

Study limitations included lack of validation, recall bias, and the lack of definition of survey items in true economic value terms; several focused independently on cost or benefit, making value interpretation more difficult.

Despite these limitations, the findings contribute to the existing literature describing the influence that the clinical learning environment has on trainee practice behaviors. Sedrak et al. showed that low-value services provided by residents were driven by the lack of high-value faculty role modeling and perceived expectations that thoroughness is valued over restraint. ${ }^{2}$ Ryskina et al. showed that faculty discussions about high-value care during patient care rounds increased trainee self-reported high-value practices. ${ }^{3}$

In 1975, Hiatt argued that physicians are both advocates for the patient and stewards of the medical commons and implied that physicians would not be making the US's critical health care decisions alone. ${ }^{4}$ Unfortunately, the tragedy of the medical commons in the USA has been growing ever since, prompting Cooke in 2010 to suggest that one reason for this problem is that the culture of traditional academic medicine has relegated education about high-value care to a lower state of intellectualization. Cooke subsequently questioned whether today's medical education can prepare the next generation to participate in national health care decision-making at all. ${ }^{5}$ The conclusions of Hunderfund et al., when combined with the preceding relevant literature, should reduce any doubt that attending physicians exert significant influence over the value of health care provided by medical trainees. The conclusions also suggest that the answer to Cooke's important question lies less in the ability to directly educate trainees about high-value care and more in the ability to drive high-value adaptive change to the practice behaviors of the current generation of attending physicians.

Corresponding Author: Daniel G. Hoody, MD, MS; Academic Hospital, Hennepin Healthcare, Minneapolis, MN, USA (e-mail: Daniel.hoody@hcmed.org).

\section{Compliance with Ethical Standards:}

Conflict of Interest: The author declares that he does not have a conflict of interest.

\section{REFERENCES}

1. Hunderfund ANL, Starr SR, Dyrbye LN, Baxley EG, Gonzalo JD, Miller BM, George P, Morgan HK, Allen BL, Hoffman A, Fancher TL, Mandrekar J, Reed DA. Imprinting on Clinical Rotations: Multisite Survey of High- and Low-Value Medical Student Behaviors and Relationship with Healthcare Intensity. J Gen Intern Med. https://doi.org/10.1007/s11606019-04828-8.

2. Sedrak MS, et al. Residents' self-report on why they order perceived unnecessary inpatient laboratory tests. J Hosp Med. 2016;11(12):869-872.

3. Ryskina KL, et al. U.S. Internal Medicine Residents' Knowledge and Practice of High-Value Care: A National Survey. Acad Med. 2015;90(10):1373-9.

4. Hiatt HH. Protecting the medical commons: who is responsible? N Engl $J$ Med. 1975;293(5):235-41.

5. Cooke M. Cost consciousness in patient care-what is medical education's responsibility? N Engl J Med. 2010;362(14):1253-5.

Publisher's Note Springer Nature remains neutral with regard to jurisdictional claims in published maps and institutional affiliations.

Published online April 22, 2019 